\title{
Enormous shrinkage of carbon nanotubes by supersonic stress and low-acceleration electron beam irradiation
}

\author{
Jun-ichi Fujita, ${ }^{\text {a) }}$ Teppei Takahashi, and Ryuichi Ueki \\ University of Tsukuba, Institute of Applied Physics, Tsukuba 305-8573, Japan
}

Takeshi Hikata and Soichiro Okubo

Sumitomo Electric Industries, Ltd., Konohana-ku, Osaka 554-0024, Japan

Risa Utsunomiya and Teruaki Matsuba

Nissin Electric Co., Ltd., Ukyo-ku, Kyoto 615-8686, Japan

(Received 4 December 2011; accepted 11 February 2012; published 15 March 2012)

\begin{abstract}
The authors demonstrated a new method for inducing enormous shrinkage in single-walled carbon nanotube bundles by applying low energy electron beam irradiation along with supersonic vibration, and a maximum shrinkage rate of $-100 \% \mathrm{~cm}^{2} / \mathrm{C}$ was obtained under electron acceleration of $1 \mathrm{keV}$. The characteristic feature of the shrunken single-walled carbon nanotubes was a wavy deformation that affected the entire bundle. The authors believe that a uniaxial stress induced by the supersonic vibration broke the equilibrium of the internal stress and allowed the uniform accumulation of defects under low energy electron beam excitation. The wavy deformation of the single-walled carbon nanotubes resulted in the enormous shrinkage of the bundle. (C) 2012 American Vacuum Society. [http://dx.doi.org/10.1116/1.3694027]
\end{abstract}

\section{INTRODUCTION}

Supercapacitors have become a key energy storage device for quickly supplying a huge current $\operatorname{load}^{1-3}$ for application in both future smart grids and hybrid automobile engines. The basic principle is very simple: An extremely large surface area exceeding about $\sim 1000 \mathrm{~m}^{2} / \mathrm{g}$, which is achieved using an electrical double layer, inevitably results in a very high capacitance. ${ }^{4,5}$ For single-walled carbon nanotubes (SWCNTs), a substantial nanotube surface area per unit volume can be automatically determined if both the diameter and the packing density of the nanotubes are specified. Therefore, the artificial decoration of the SWCNT surface with a functional group ${ }^{6,7}$ drastically increases the surface area, up to $1300 \mathrm{~m}^{2} / \mathrm{g}$, and the performance (energy density of $94 \mathrm{Wh} / \mathrm{kg}$ and power density of $210 \mathrm{~kW} / \mathrm{kg}$ ) almost reaches their ideal values. ${ }^{8}$ Despite such superior characteristics of a CNT-based supercapacitor, further improvement of its storage density is required for practical applications such as electrical vehicles.

An interesting method for overcoming such storage capacity limitation involves the creation of wormholes on the CNT body using chemical etching and zipping. This enlarges the effective area for the electrical double layer. ${ }^{9}$ In contrast, our method involves increasing the surface area by introducing a bellowslike wrinkle structure into the CNT body based on an electron-induced reaction with surface stress. ${ }^{10}$

It is well known that high energy electron irradiation induces a variety of structural changes in carbon nanotubes; perpendicularly incident electrons induce lateral ejection of carbon atoms; thus creating Stone-Wales (SW) defects on the honeycomb lattice. ${ }^{1-16}$ The threshold energy needed to induce this so-called "knock-on mechanism" is about $86 \mathrm{keV}, 11$

$\overline{{ }^{a} \text { Electronic mail: fujita@bk.tsukuba.ac.jp }}$ and the propagation of the SW defect plays an essential role in many structural transformations, ${ }^{13}$ because a heptagonheptagon combination cannot sustain the initial flat surface but instead generates a local curvature. Therefore, SW defects combined with local excitation of the carbon bond induces a variety of carbon nanostructures ${ }^{11,17-21}$ including the creation of fullerenes from carbon soot, ${ }^{17}$ lattice destruction into amorphous carbon, ${ }^{11,18}$ nanotube scissoring, ${ }^{19}$ and the cross-linking of nanotubes and fullerenes. ${ }^{20,21}$

On the other hand, low energy electrons are considered to be free from producing irradiation damage when the energy is lower than the knock-on threshold. However, because the low energy electrons also induce scissoring ${ }^{22,23}$ and CNT cross-linking; ${ }^{24,25}$ significant damage is induced even when the electron energy is reduced to about $1 \mathrm{keV} .{ }^{25-27}$ The incident low energy electrons induce multiple energy transfers that coincide with bond excitation and the subsequent highly excited state, which is equivalent to local heating, leads to subsequent molecular destruction ${ }^{28}$ producing the formation of defects that induce nanotube cutting and welding.

We previously reported that a CNT exhibits extreme shrinkage under low energy electron irradiation with the assistance of a slight tensile stress, where the maximum shrinkage was about $90 \%$ against the initial length. ${ }^{10}$ Here, a single nanotube fiber was suspended between an AFM cantilever and a piezoactuated probe under an electron beam imaging field. While the suspended nanotube failed to shrink under stress-free exposure, the application of tensile stress, even an extremely small stress of less than $10 \mathrm{nN}$, induced remarkable shrinkage of the nanotube to about one-tenth of its original length under an electron dose of about $10 \mathrm{C} / \mathrm{cm}^{2}$. This shrunken CNT, having a bellowlike surface corrugation, showed a slight increase in diameter. Thus, the effective multiplication factor for the surface increase per unit volume was about 6. 
Also seen in this pervious study, the characteristic feature of the shrunken multiwalled nanotube (MWNT) was that its multiwalled body was transformed into a bellowlike crushed structure. A transmission electron microscopy (TEM) image indicated that the tube was not destroyed nor changed into an amorphous one. Instead, the tube body maintained its honeycomb-based structure with zigzag folding. In addition, the circular cross-section of the shrunken MWNT was highly deformed to form a polygonal shape. Thus, the application of tensile stress effectively induced the axial shrinkage of the nanotube. However, such one-by-one manipulation of a CNT fiber is state-of-the-art nanotechnology and it requires crucial improvements for mass production.

In this paper, we report our new method for effectively inducing CNT shrinkage using internal stress induced by supersonic vibrations. We report the details of supersonicinduced CNT shrinkage.

\section{EXPERIMENT}

We built an in situ experimental setup where a singlewalled carbon nanotube (SWNT) bundle was placed on the edge of a specimen holder (copper mesh) and a piezo oscillator was mounted on the backside of the holder assembly, as shown in Fig. 1. The CNT specimen was heated to about $100{ }^{\circ} \mathrm{C}$ to reduce carbon contamination. All the experiments were carried out inside the specimen chamber of a scanning electron microscope (Hitachi S4800), where the typical back pressure was lower than $10^{-3} \mathrm{~Pa}$. The supersonic vibration induced vibrations in the free-standing SWNT bundle just supported at the edge of the copper mesh. Thus, a periodic tensile and compression stress was induced in the SWNT bundle.

All the experiments were carried out in an in situ imaging environment, typically with a magnification of 50000 times, using beam currents of $10 \mathrm{pA}$ at $1 \mathrm{KeV}, 30 \mathrm{pA}$ at $10 \mathrm{keV}$, and $100 \mathrm{pA}$ at $30 \mathrm{keV}$. Using this experimental setup, we evaluated the dependence of the shrinkage of the SWNT bundle upon the electron dose, applied frequency, and acceleration voltage. We also evaluated the microscopic structure of the SWNT bundle before and after shrinkage using TEM (Jeol JEM2100).

\section{RESULTS AND DISCUSSION}

Figure 2 shows a sequential snapshot of SWNT shrinkage relative to electron dose at an irradiated electron energy of

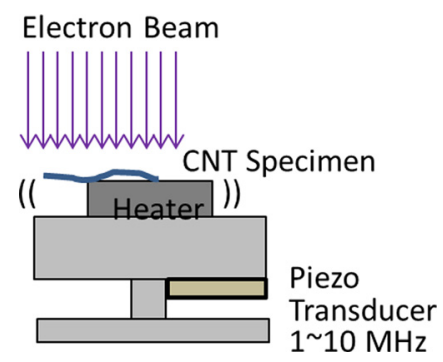

FIG. 1. (Color online) Schematic of electron shower irradiation of a carbon nanotube under supersonic vibration. A typical SWNT had a diameter of $2 \mathrm{~nm}$ and length of $1 \mu \mathrm{m}$, and the MWNT had a thickness of about $10 \mathrm{~nm}$ and a length of $1 \mu \mathrm{m}$.
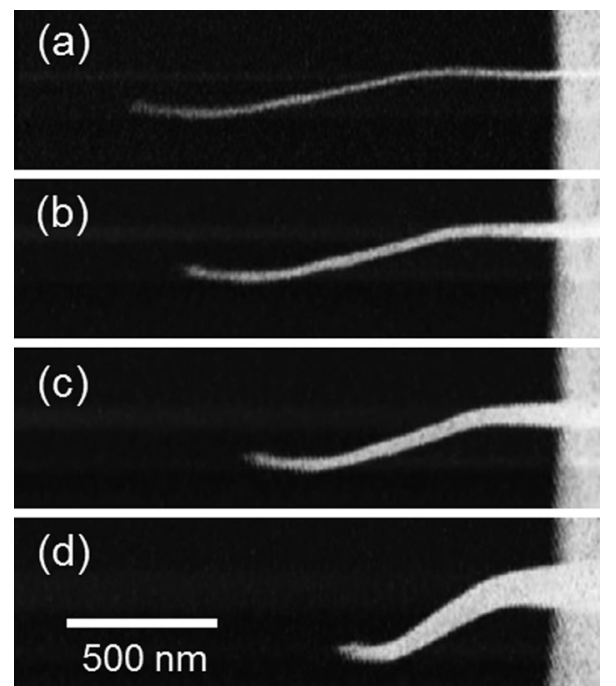

FIG. 2. Sequential snapshot during shrinkage of a SWNT bundle; (a) initial SWNT, and SEM image of a SWNT after being irradiated with electron doses of (b) $0.2 \mathrm{C} / \mathrm{cm}^{2}$, (c) $0.5 \mathrm{C} / \mathrm{cm}^{2}$, and (d) $1.0 \mathrm{C} / \mathrm{cm}^{2}$.

$5 \mathrm{keV}$. We determined the shrinkage based on the SEM image of the shrunken SWNT. The shrunken SWNT was buried in the contamination layer but fortunately the thickness of the contamination layer at the top of the bundle was not so thick, being about several tenth nanometers. Thus the measuring of the shrunken SWNT bundle contains negligible error due to contamination. The SWNT bundle monotonically shrunk with an increase in the electron dose; however, a long irradiation time simultaneously increased the carbon contamination even if the specimen was heated to $100{ }^{\circ} \mathrm{C}$. Thus, the shrinkage gradually became saturated as a tradeoff with an increase in the contamination, as shown in Fig. 3. Such carbon contamination severely affected the shape of the specimen, particularly at a low acceleration condition where the cross-section of the reaction became prominent in the decomposition of the adsorbed hydrocarbon molecules in the experimental chamber.

The shrinkage also showed a strong dependency upon the applied frequency, as shown in Fig. 4. Here, the shrinkage rate is defined as the tangential slope at the initial shrinkage per unit of electron dose, as shown by the dotted line in

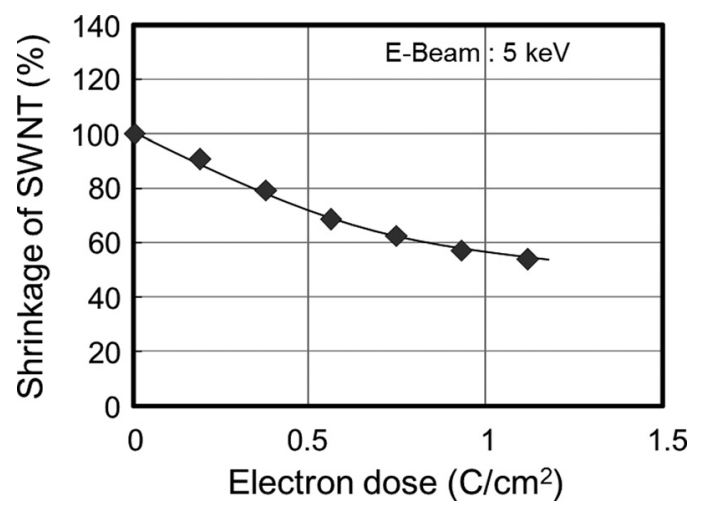

FIG. 3. Shrinkage of CNT under electron irradiation at $5 \mathrm{keV}$, and typical SEM images of carbon nanotubes before and after electron irradiation. 


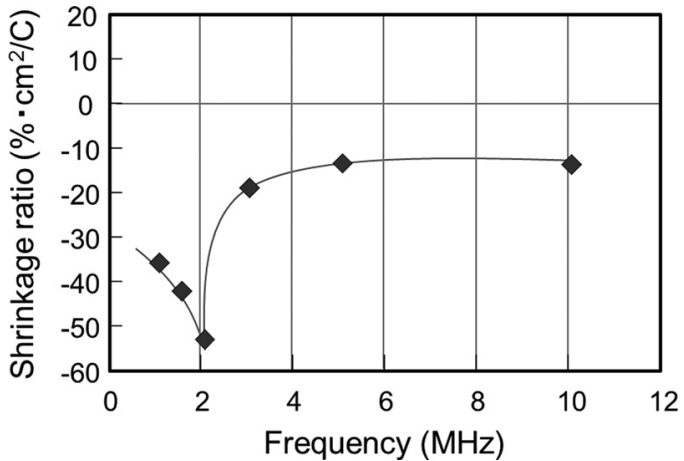

FIG. 4. Highly sensitive dependence of shrinkage rate of CNT upon the applied supersonic frequency.

Fig. 3, in order to eliminate the reduction rate effect of carbon contamination. These experiments were performed by choosing SWNT bundles with similar sizes (approximately $2-\mu \mathrm{m}$ long and 20-nm wide). The frequency of about $2 \mathrm{MHz}$ was almost identical to the first order resonant frequency of the SWNT specimen. We confirmed that the SWNT bundle had a Young's modulus of about $100 \mathrm{GPa}$, which agreed well with other experimental results. ${ }^{29,30}$ By using the Young's modulus, a resonant frequency of about $2.3 \mathrm{MHz}$ was calculated under the assumption that the SWNT bundle had a cylindrical and uniform shape with SWNT packing as follows: $f=\left(\beta^{2} / 2 \pi L\right) \sqrt{E I / \rho A}$. Here, $\beta=1.875$, and represents the 0th order of vibration; the bundle length, $L(=2 \mu \mathrm{m})$, represents the free standing region; the Young's modulus, $E$, is $100 \mathrm{GPa}$; the density is $2130 \mathrm{~kg} / \mathrm{m}^{3} ;{ }^{31}$ the geometrical moment of inertia, $I$, is $4.9 \times 10^{-34} \mathrm{~m}^{4}$; and $A$ represents the area of the cross section.

The shrinkage rate seemed to reduce rapidly when the applied frequency exceeded the resonant frequency. The amplitude of the SWNT bundle's vibration was indeed large, i.e., of the order of $100 \mathrm{~nm}$, which was detected photographically under the imaging field. The vibration induced by a frequency lower than the resonance still had a relatively large amplitude with translating motion and; thus, the shrinkage rate retained a large value. However, the vibrating amplitude was reduced drastically at a higher frequency following a simple response equation for cantilever vibration where the vibration creates a certain number of nodes but reduces the vibrating amplitude. The large vibrating amplitude of the SWNT bundle, and thus its large bending, seemed to produce the large shrinkage rate.

A resonant frequency of $2 \mathrm{MHz}$ was found to give the maximum shrinkage. However, the reduction in the length; in addition to the wearing due to carbon contamination during beam irradiation, should increase the resonant frequency. This resonant frequency shift could be identical to the forced vibration condition under a frequency lower than the resonant frequency.

The strain and stress distribution should be minimized at the free end of the bundle and; therefore, the top end of the bundle should remain free from shrinkage if the vibration is maintained at resonance. However, continuous shrinkage soon deviated from resonance conditions because the oscillating

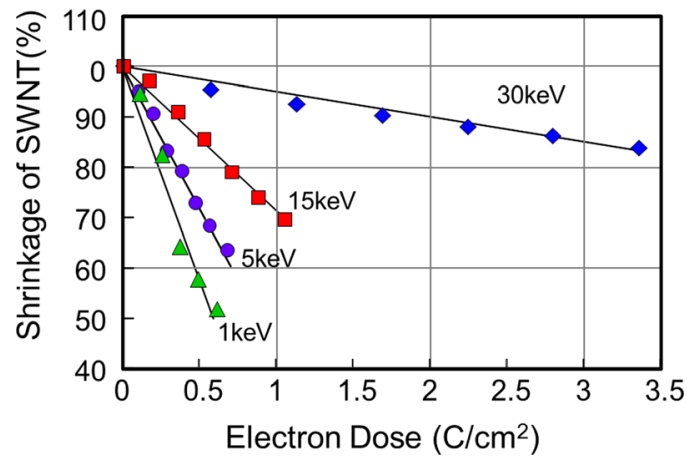

FIG. 5. (Color online) Shrinkage rate dependency upon electron dose. Reducing the electron energy drastically increased the shrinkage rate, as indicated by the slope.

frequency was kept constant and; thus, the position of nodes and loops always moved on the vibrating bundle. Therefore, we think there is no single point of shrinkage in the bundle.

The shrinkage of the SWNT bundle always coexisted with carbon contamination, in particular at lower electron energies of around $1 \mathrm{keV}$. Such encasing of amorphous carbon presumably induced a certain amount of compressive stress which may affect the manner of shrinkage. However, we clearly observed the frequency-dependent shrinkage, where the data were taken under the same electron dose, under contamination conditions. Therefore we think that the shrinkage of the SWNT was induced by an intrinsic structure change under low energy electron irradiation.

Reducing the acceleration voltage strongly enhanced the SWNT shrinkage, as shown in Fig. 5, and the acceleration voltage dependencies are shown in Fig. 6. The CNT shrinkage was very sensitive to the acceleration voltage, with the shrinkage maxima observed at about $1 \mathrm{keV}$; yet, the maximum shrinkage seemed to be realized in a much lower acceleration voltage regime. In addition, the experimental error was widely distributed from $-35 \% \mathrm{~cm}^{2} / \mathrm{C}$ to $-100 \% \mathrm{~cm}^{2} / \mathrm{C}$ at the acceleration voltage of $1 \mathrm{keV}$, which is represented by the error bar in Fig. 6.

The shrinkage at the low acceleration voltage was strongly influenced by carbon contamination, even though

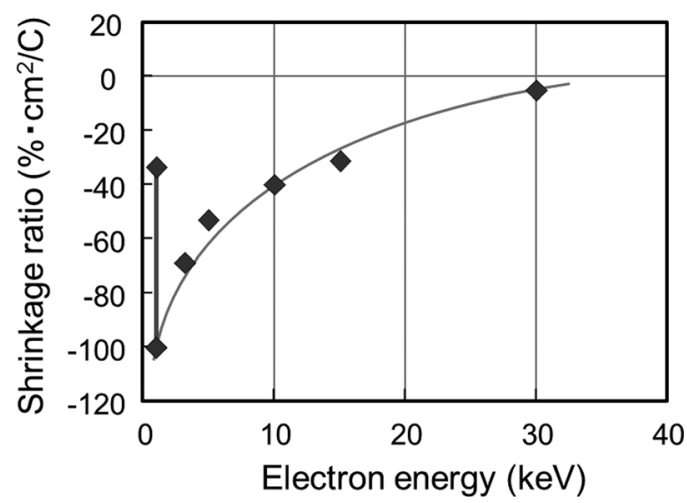

FIG. 6. Shrinkage rate dependency of CNT upon the irradiated electron energy. The error in the data at $1 \mathrm{keV}$ was widely distributed because of severe carbon contamination, but the maximum shrinkage rate was obtained. 
the experiment was carried out at $100^{\circ} \mathrm{C}$. However, the shrinkage rate for each acceleration voltage seemed to be inversely proportional to the voltage, with a shrinkage rate of about $-40 \%$ at $10 \mathrm{keV}$ reduced to about $-5 \%$ at $30 \mathrm{keV}$, if we exclude the shrinkage data obtained at values lower than $10 \mathrm{keV}$ in order to avoid the serious influence from carbon contamination. This acceleration voltage dependency presumably reflects the cross-section of the electron for the bond excitation under a simple energy transfer scheme of electron scattering.

The shrinkage of an MWNT in our previous experiment resulted in an accordion like folding, ${ }^{10}$ and such shrinkage structures were frequently observed in a relatively thick MWNT of about $20 \mathrm{~nm}$. However, the width of the SWNT was very small (about $2 \mathrm{~nm}$ ) and; thus, the SWNT could not form such an accordionlike corrugation, but instead formed a wavy deformation that extended into the entire bundle. Thus, the lattice image of the shrunken SWNT bundle contains a clearly contrasted area sandwiched by periodic blurry regions, indicated by white arrows in Fig. 7(b).

The SWNTs in the bundle were aggregated by the van der Waals force and; thus, the coherent deformation of the SWNT, rather than random deformation, could minimize the surface energy. The projected lattice image of the SWNT bundle was characterized by a very straight and parallel contrast along the tube axis coinciding with the periodic blur regions. All of the lattice images suggested

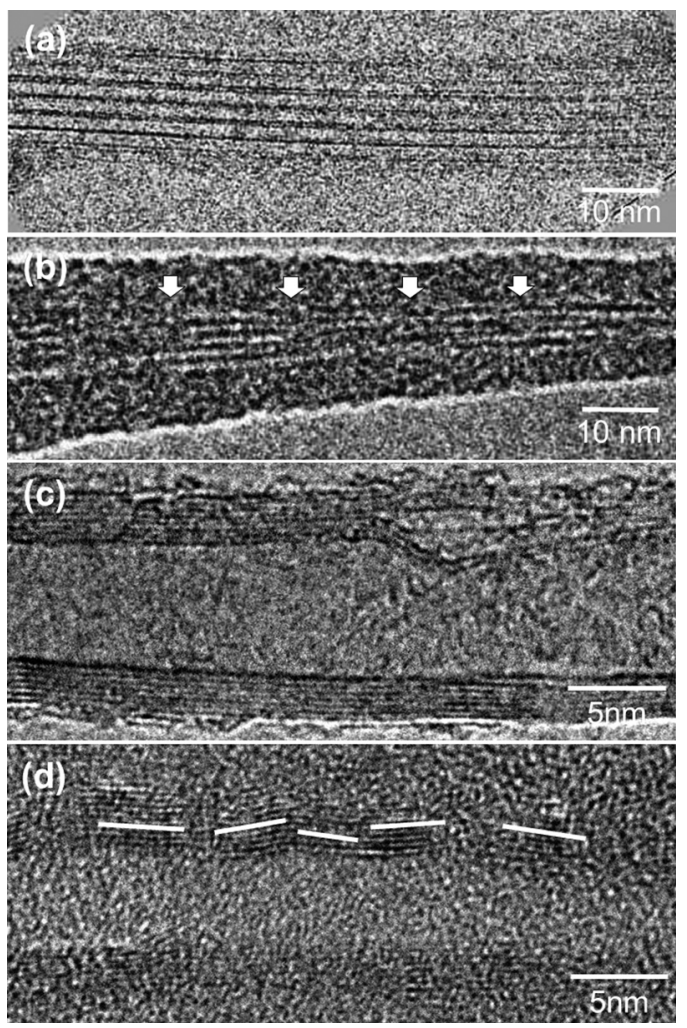

FIG. 7. TEM images of (a) initial SWNT bundle, (b) shrunken SWNT bundle by $1 \mathrm{keV}$ electron irradiation, (c) initial MWNT, and (d) shrunken MWNT by $1 \mathrm{keV}$ electron irradiation. The arrows suggest the positions of periodic blur in (b) and solid lines in (d) are guides of the zigzag folding in MWNT. that the deformation never included the zigzag wrinkling that was observed in the MWNT, but was rather a gentle wavy deformation. A slight change in the depth of focus for the TEM imaging shifted the blur region so as to keep the continuity of the projected tube image, which suggested that the shrunken SWNT had a geometrically wavy and/or twisting deformation.

While the linear alignment of the SW defects generated wrinkle ridges, ${ }^{16}$ the relatively small curvature of the SWNT body had a small tolerance to the introduction of wrinkle ridges into its body because the transformation energy needed to introduce a large folding angle into the thin SWNT wall would be extremely large; much more than needed for the destruction of the honeycomb lattice. However, the van der Waals attraction helped to preserve the relative interspacing of the tubes from the destruction of the SWNT bundle structure. Thus, when the SWNT shrunk it had a wavy deformation where the internal stress was periodically distributed to the wall so as to minimize the surface stress.

It is well known that the generation of an SW defect consumes about $5 \mathrm{eV}$ to eject a knock-on carbon atom from the honeycomb lattice, ${ }^{13,14}$ where the transferred energy is almost identical to the approximate $80 \mathrm{keV}$ for the perpendicularly irradiated electron beam. However, the electron energy that induced the tube shrinkage was much smaller than the threshold value, and the largest shrinkage rate was observed at $1 \mathrm{keV}$ in this experiment. In addition, the larger shrinkage rate seemed to be realized at a lower electron energy, while the severe carbon contamination seemed to tradeoff the shrinkage.

Although the direct creation of SW defects did not occur in our experiment using low energy electron irradiation, the SW defects seem to be generated through a local reorganization process between the $\mathrm{sp}^{2}$ and $\mathrm{sp}^{3}$ excited states if we take into account the fact that tube scissoring and cross-linking are reported under almost the same electron energy regime, along with the fact that defect creation under $1 \mathrm{keV}$ of electron irradiation was suggested through Raman spectroscopy measurements. ${ }^{27}$ Although all of the previously described shrinkage data were based on SWNT bundles, we also confirmed that the supersonic vibration effectively induced the shrinkage of MWNTs, as shown in the TEM images of MWNTs before and after electron shower irradiation. The shrunken MWNT under lower energy electron irradiation was characterized by zigzag folding of the basal plane like an accordion wall, which was essentially the same structure that we observed in the shrinkage under a uniaxial tensile stress. ${ }^{10}$

We believe that the low energy electron irradiation may induce bond excitation in the $\mathrm{sp}^{2}$ hexagonal network, and that the reorganization process for the excited states among $\mathrm{sp}^{2}$, $\mathrm{sp}^{2+\delta}$, and $\mathrm{sp}^{3}$ generates defects and certain bond angles. ${ }^{28}$ Such an excitation was stochastic and randomly dispersed in the wall. Thus, all of the internal stress was compensated unless external stress was applied. However, the supersonic vibration induced a periodic and uniaxial compressive and tensile stress and; thus, the equilibrium of the internal stress 
was broken and deformation started to minimize the lattice distortion with defects and bond angles.

\section{SUMMARY AND CONCLUSIONS}

We demonstrated a new method to induce enormous shrinkage in SWNTs and MWNTs through the application of low energy electron beam irradiation combined with supersonic vibration, where the maximum shrinkage rate of $-100 \% \mathrm{~cm}^{2} / \mathrm{C}$ was obtained under an electron acceleration of $1 \mathrm{keV}$.

We believe that the low energy electron irradiation induced random excitation in the $\mathrm{sp}^{2}$ honeycomb lattice and created defects with certain bond angles. At the same time, such a stochastic and randomly dispersed excitation resulted in the equilibrium of the internal stress unless an external stress was applied. Thus, breaking this equilibrium with a uniaxial stress would be the main role of the supersonic vibration to induce the enormous nanotube shrinkage.

Although further improvement is needed for the mass production of shrunken carbon nanotubes, our shrinkage technique would be very useful for a nanotube-based energy storage device, particularly as the electrode of a supercapacitor device.

\section{ACKNOWLEDGMENT}

This work was supported by a Grant-in-Aid for Scientific Research (Grant No. 23246063).

${ }^{1}$ C. Niu, E. K. Sichel, R. Hoch, D. Moy, and H. Tennent, Appl. Phys. Lett. 70, 1480 (1997)

${ }^{2}$ R. Z. Ma, J. Liang, B. Q. Wei, B. Zhang, C. L. Xu, and D. H. Wu, J. Power Sources 84, 126 (1999).

${ }^{3}$ K. H. An, W. S. Kim, Y. S. Park, J. M. Moon, D. J. Bae, S. C. Lim, Y. S. Lee, and Y. H. Lee, Adv. Funct. Mater. 11, 387 (2001).

${ }^{4}$ T. Hiraoka et al., Adv. Funct. Mater. 20, 422 (2010).
${ }^{5}$ A. Peigney, C. Laurent, E. Flahaut, R. R. Bacsa, and A. Rousset, Carbon 39, 507 (2001).

${ }^{6}$ Q. Xiao and X. Zhou, Electrochim. Acta. 48, 575 (2003).

${ }^{7}$ H. Zhang, G. Cao, Z. Wang, Y. Yang, Z. Shi, and Z. Gu, Nano Lett. 8, 2664 (2008).

${ }^{8}$ A. I. Najafabadi, S. Yasuda, K. Kobashi, T. Yamada, D. N. Futaba, H. Hatori, M. Yumura, S. Iijima, and K. Hata, Adv. Mater. 22, E235 (2010).

${ }^{9}$ D. N. Futaba et al., Nat. Mater. 5, 987 (2006).

${ }^{10}$ R. Ueki, T. Hikata, S. Ookubo, R. Utsunomiya, T. Matsuba, and J. Fujita, Jpn. J. Appl. Phys. 50, 06GE10 (2011).

${ }^{11}$ B. W. Smith and D. E. Luzzia, J. Appl. Phys. 90, 3509 (2001).

${ }^{12}$ A. Zobelli, A. Gloter, C. P. Ewels, G. Seifert, and C. Colliex, Phys. Rev. B 75, 245402 (2007)

${ }^{13}$ J. Ma, D. Alfè, A. Michaelides, and E. Wang, Phys. Rev. B 80, 033407 (2009).

${ }^{14}$ L. G. Zhou and S. Shi, Appl. Phys. Lett. 83, 1222 (2003).

${ }^{15}$ J. Kotakoski, J. C. Meyer, S. Kurasch, D. Santos-Cottin, U. Kaiser, and A. V. Krasheninnikov, Phys. Rev. B 83, 245420 (2011).

${ }^{16}$ M. T. Lusk and L. D. Carr, Phys. Rev. Lett. 100, 175503 (2008).

${ }^{17}$ T. F'tiller and F. Banhart, Chem. Phys. Lett. 254, 372 (1996).

${ }^{18}$ P. M. Ajayan, V. Ravikumar, and J. C. Charlier, Phys. Rev. Lett. 81, 1437 (1998).

${ }^{19}$ F. Banhart, J. Mater. Sci. 41, 4505 (2006).

${ }^{20}$ M. Terrones, F. Banhart, N. Grobert, J.-C. Charlier, H. Terrones, and P. M. Ajayan, Phys. Rev. Lett. 89, 075505 (2002).

${ }^{21}$ D. Ugarte, Nature 359, 707 (1992).

${ }^{22}$ T. D. Yuzvinsky, A. M. Fennimore, W. Mickelson, C. Esquivias, and A. Zettl, Appl. Phys. Lett. 86, 053109 (2005).

${ }^{23}$ G. Y. Gerasimov, J. Eng. Phys. Thermophys. 83, 849 (2010).

${ }^{24}$ F. Banhart, Nano Lett. 1, 329 (2001).

${ }^{25}$ C. K. Harnett, K. M. Satyalakshmi, and H. G. Craighead, Appl. Phys. Lett. 76, 2466 (2000)

${ }^{26}$ S. Suzuki and Y. Kobayashi, Chem. Phys. Lett. 430, 370 (2006).

${ }^{27}$ S. Suzuki, K. Kanzaki, Y. Homma, and S. Fukuba, Jpn. J. Appl. Phys. 43, L1118 (2004).

${ }^{28}$ S. Gupta, R. J. Patela, N. Smith, R. E. Giedd, and D. Hui, Diamond Relat. Mater. 16, 236 (2007).

${ }^{29}$ J. P. Salvetat, G. Andrew, D. Briggs, J. M. Bonard, R. R. Bacsa, and A. J. Kulik, Phys. Rev. Lett. 82, 944 (1999).

${ }^{30}$ J. P. Lu, Phys. Rev. Lett. 79, 1297 (1997).

${ }^{31}$ Q. Lu, G. Keskar, R. Ciocan, R. Rao, R. B. Mathur, A. M. Rao, and L. L. Larcom, J. Phys. Chem. B 110, 24371 (2006). 